\title{
Drug treatment for oral submucous fibrosis
}

\author{
Abstracted from \\ Jiang $X$, Hu J. \\ Drug treatment of oral submucous fibrosis: a review of the literature. \\ J Oral Maxillofac Surg 2009; 67: 1510-1515. \\ Address for correspondence: $\mathrm{Dr} J \mathrm{Hu}$, Department of Oral and Maxillofacial Surgery, \\ West China College of Stomatology, Sichuan University, Chengdu 610041, China. E-mail: drhu@vip.sohu.com
}

\section{Question: What drugs can be used to treat oral submucous fibrosis?}

\begin{abstract}
Data sources Searches were made for relevant data using Medline and the International Poster Journal of Dentistry and Oral Medicine online database (see www.ipj.quintessenz.de).

Study selection Meta-analyses, randomised controlled trials (RCT), clinical trials and other experimental designs were considered. Data extraction and synthesis Because of heterogeneity of study designs and drugs used, a qualitative synthesis was conducted. Results Seventeen publications were identified of which 15 were included. Of these, six were RCT, four were clinical trials/ controlled clinical trials, and five were other types experimental studies. The studies in total involved 1224 patients. The rate of those lost to followup reached $30 \%$ in some studies. The drugs used to treat oral submucous fibrosis (OSF) were categorised into steroids, enzymes, cardiovascular drugs, antioxidants, vitamins and microelements. Conclusions There are few high-quality studies available and the present drug treatments are in general empirical and treat only symptoms. There is a need for high-quality RCT in this area, especially studies involving combined and sequential therapy.
\end{abstract}

\section{Commentary}

OSF is a chronic progressive scarring oral disease typically affecting Asians. It is characterised by a burning sensation, blanching and stiffening of the oral mucosa and, in the advanced stages, the jaws may become inseparable with resultant difficulty in eating, swallowing, speech and maintenance of oral health. What makes it more sinister is its potential for malignant transformation, with the reported risk ranging from $2.3-7.6 \% .^{1,2}$

Scientific literature to date provides firm evidence that areca nut use is the major aetiological factor but the exact mechanism of areca nut constituents' action on the oral mucosal tissue remains unknown. Nevertheless, most studies propose increased collagen synthesis and reduced degradation by various pathways as the likely pathogenesis. This knowledge has enabled the development of numerous therapeutic strategies which are broadly categorised into surgical and nonsurgical (ie, drugs and physiotherapy) approaches and are employed according to the severity of lesions. ${ }^{3}$

This review focuses on the drug management of this enigmatic disease and has identified all the pertinent studies. The drugs used to date includes steroids, fibrinolytic enzymes, anti-ischemic vasodilators, anti-oxidants, nutritional supplements, anti-fibrotic agents and biogenic stimulants, all of which have delivered variable results. Most drugs have shown quick relief of symptoms, namely the burning sensation and ulceration, reducing the stiffness of the mucosa to a certain extent. None of them, however, has demonstrated any marked improvement in the trismus, signifying that drugs in OSF serve merely as adjuncts; being useful only in the early stages of disease.

The review thus fails to answer the question of what drugs are appropriate for the management of OSF, and correctly states that none of the drugs discussed have provided satisfactory results. There are numerous reasons for this outcome, amongst them: obscure pathogenesis, lack of adequate followup, deficient co-operation from patients in giving up their areca nut habits, insufficient medical resources in developing countries where OSF is more prevalent, high incidence of relapse, presence of side-effects, poor patient compliance and, importantly, the lack of well-designed and meticulous clinical trials. Consequently, vigorous clinical research is still required to provide the evidence that would enable clinicians to make objective decisions over the effectiveness of the available treatment options. Additionally, educational interventions regarding the ill effects of areca nut use and application of more stringent laws to impede marketing of areca nut products could assist in addressing this public health issue.

Punnya V Angadi

Department of Oral and Maxillofacial Pathology, KLE VK Institute of Dental Sciences and Hospital, Belgaum,

Karnataka, India

1. Murti PR, Bhonsle RB, Pindborg IJ, Daftary DK, Gupta PC, Mehta FS. Malignant transformation rate in oral submucous fibrosis over a 17 year period. Community Dent Oral Epidemiol 1997; 25: 377-383.

2. Tilakaratne WM, Klinikowski MF, Takashi S, Peters TJ, Warnakulasuriya S. Oral submucous fibrosis: review on etiology and pathogenesis. Oral Oncol 2006; 42: 561-568.

3. Khanna JN, Andrade NN. Oral submucous fibrosis: a new concept in surgical management. Report of 100 cases. Int J Oral Maxillofac Surg 2006; 24: 433-449.

\section{Practice points}

- The difficulty faced by practitioners in the management of OSF may be countered by obtaining the right combination of drugs and surgery in individual cases. 